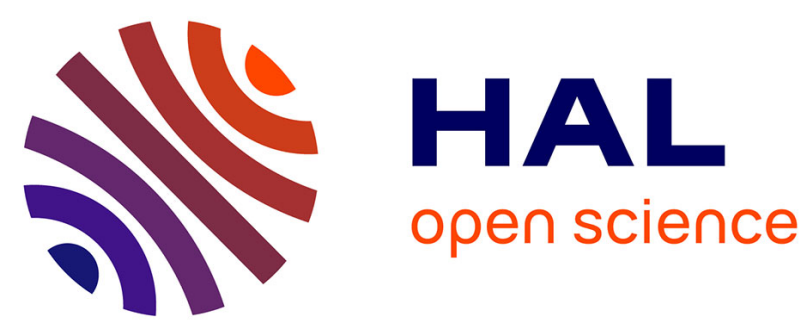

\title{
Performance comparison of Bayesian iterative algorithms for three classes of sparsity enforcing priors with application in computed tomography
}

\author{
Mircea Dumitru, Wang Li, Nicolas Gac, Ali Mohammad-Djafari
}

\section{To cite this version:}

Mircea Dumitru, Wang Li, Nicolas Gac, Ali Mohammad-Djafari. Performance comparison of Bayesian iterative algorithms for three classes of sparsity enforcing priors with application in computed tomography. 2017 IEEE International Conference on Image Processing, Sep 2017, Beijing, China. hal01568337

\section{HAL Id: hal-01568337 \\ https://hal.science/hal-01568337}

Submitted on 25 Jul 2017

HAL is a multi-disciplinary open access archive for the deposit and dissemination of scientific research documents, whether they are published or not. The documents may come from teaching and research institutions in France or abroad, or from public or private research centers.
L'archive ouverte pluridisciplinaire $\mathbf{H A L}$, est destinée au dépôt et à la diffusion de documents scientifiques de niveau recherche, publiés ou non, émanant des établissements d'enseignement et de recherche français ou étrangers, des laboratoires publics ou privés. 


\title{
PERFORMANCE COMPARISON OF BAYESIAN ITERATIVE ALGORITHMS FOR THREE CLASSES OF SPARSITY ENFORCING PRIORS WITH APPLICATION IN COMPUTED TOMOGRAPHY
}

\author{
Mircea Dumitru $\quad$ Li Wang ${ }^{\star} \quad$ Nicolas Gac $\quad$ Ali Mohammad-Djafari ${ }^{\star}$ \\ ${ }^{\star}$ Laboratoire des signaux et systèmes, CentraleSupélec, Gif-sur-Yvette, 91192, France
}

\begin{abstract}
The piecewise constant or homogeneous image reconstruction in the context of X-ray Computed Tomography is considered within a Bayesian approach. More precisely, the sparse transformation of such images is modelled with heavy tailed distributions expressed as Normal variance mixtures marginals. The derived iterative algorithms (via Joint Maximum A Posteriori) have identical updating expressions, except for the estimated variances. We show that the behaviour of the each algorithm is different in terms of sensibility to the model selection and reconstruction performances when applied in Computed Tomography.
\end{abstract}

Index Terms - Normal variance mixtures, Bayesian inference, sparsity enforcing priors, image reconstruction.

\section{INTRODUCTION}

The linear forward model considered in this paper is given by

$$
\left\{\begin{array}{l}
g=\boldsymbol{H} \boldsymbol{f}+\boldsymbol{\epsilon} \\
\boldsymbol{f}=\boldsymbol{D} \boldsymbol{z}+\boldsymbol{\xi}
\end{array},\right.
$$

where $\boldsymbol{g}$ represents the observed data, $\boldsymbol{H}$ represents the linear projection operator, $\boldsymbol{f}$ represents the image to be reconstructed, $\epsilon$ accounts for errors and model uncertainties [15]. The piecewise continuous image $f$ is expressed via a transformation $\boldsymbol{D}$ (such as Haar wavelet transform) of a sparse structure $z$ accounting for the uncertainties $\boldsymbol{\xi}$. The prior distribution of the sparse structure $z$ is modelled by using heavy tailed distributions expressed as marginals of $\operatorname{Normal}(\mathcal{N})$ variance mixtures [8], [2]. Conjugate priors are considered as the mixing $(\mathcal{M})$ distributions. Three categories of distributions are considered: a) Inverse Gamma $(\mathcal{I} \mathcal{G})$ distribution, (Student-t $(\mathcal{S} t)$ prior) [9], [14], [7], [4], b) a particular case of the generalized inverse Gaussian $(\mathcal{G I} \mathcal{G})$ distribution (Normal inverse Gaussian $(\mathcal{N I} \mathcal{G})$ prior) [12], [10], [11] and c) Gamma $(\mathcal{G})$ distribution (Variance-Gamma $(\mathcal{V G})$ prior) [1], [13].

Non-stationary independent Gaussian uncertainties model is assumed with conjugate priors modelling their corresponding variances. Figure (1) shows the structure of the hierarchical model and the likelihood and main prior laws proposed. In this context, regardless of the prior, the corresponding hierarchical model has the same multivariate Normal distributions assigned for $\left(\boldsymbol{g} \mid \boldsymbol{f}, \boldsymbol{v}_{\epsilon}\right),\left(\boldsymbol{f} \mid \boldsymbol{z}, \boldsymbol{v}_{\xi}\right)$ and $\left(\boldsymbol{z} \mid \boldsymbol{v}_{z}\right)$. Consequently, the posterior distribution

$$
\begin{aligned}
& p\left(\boldsymbol{f}, \boldsymbol{z}, \boldsymbol{v}_{\epsilon}, \boldsymbol{v}_{\xi}, \boldsymbol{v}_{z} \mid \boldsymbol{g}\right) \propto p\left(\boldsymbol{g} \mid \boldsymbol{f}, \boldsymbol{v}_{\epsilon}\right) p\left(\boldsymbol{f} \mid \boldsymbol{z}, \boldsymbol{v}_{\xi}\right) p\left(\boldsymbol{z} \mid \boldsymbol{v}_{z}\right) p\left(\boldsymbol{v}_{\epsilon} \mid \boldsymbol{\theta}_{\epsilon}\right) \\
& p\left(\boldsymbol{v}_{\xi} \mid \boldsymbol{\theta}_{\xi}\right) p\left(\boldsymbol{v}_{z} \mid \boldsymbol{\theta}_{z}\right) .
\end{aligned}
$$

will have a common part (formed by the product of the three multivariate Normal distributions) regardless of the prior chosen to enforce sparsity. This leads to identical analytical expressions for the estimates of $\boldsymbol{f}$ (respectively $\boldsymbol{z}$ ) when Joint Maximum A Posterior (JMAP) estimation is considered. Depending on the mixing distribution, different estimates correspond to $\boldsymbol{v}_{\epsilon}, \boldsymbol{v}_{\xi}$ and $\boldsymbol{v}_{z}$. In this paper, first the theoretical sim-

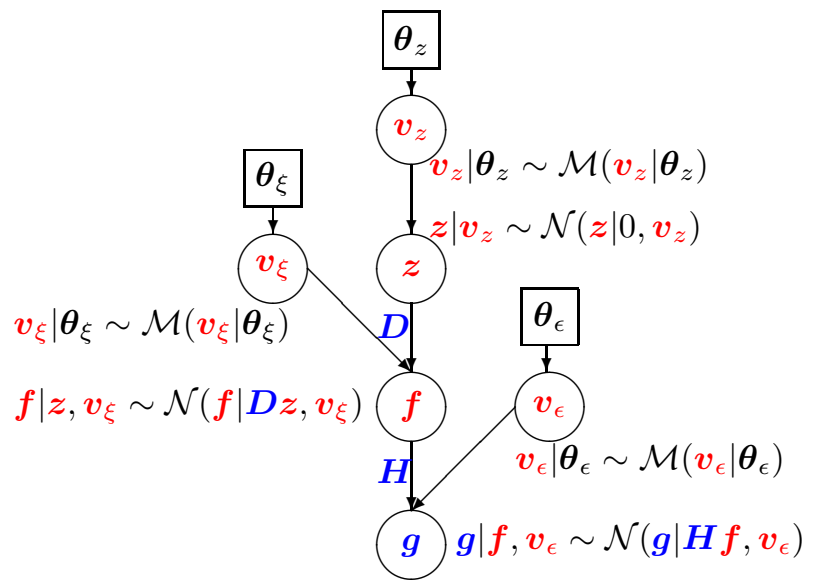

Fig. 1: Hierarchical model for forward model, Eq. (1).

ilarities and differences between the JMAP algorithms corresponding to different $\mathcal{M}$ distributions are presented. Then, a practical study of the JMAP estimation performances in the context of X-ray Computed Tomography reconstruction for different number of projections and different levels of noise. Also, we investigate the behaviour of the algorithms in terms of stability (relative to prior hyperparameters values, i.e. the mixing distribution parameters) and convergency rate.

The rest of the paper is organized as follows. Sec. (2) provides a brief presentations of the $\mathcal{M}$ distributions considered and their corresponding (heavy tailed) priors. Sec. (3) presents 
and compares the JMAP iterative algorithms corresponding the $\mathcal{M}$ distributions considered. Simulations results and empirical evaluations are presented in Sec. (4). Conclusions are drawn in Sec. (5).

\section{MIXING DISTRIBUTIONS AND PRIOR MODELS}

The sparse structure of $\boldsymbol{z}$ is accounted by a prior model using heavy-tailed distributions for each pixel $z_{j}$. We focus on such distributions that can be expressed as marginals of Normal variance mixtures. In this context, the general prior model is given by Eq. (3):

$$
\text { Prior Model: }\left\{\begin{array}{l}
p\left(z_{j} \mid 0, v_{z_{j}}\right)=\mathcal{N}\left(z_{j} \mid 0, v_{z_{j}}\right) \\
p\left(v_{z_{j}} \mid \boldsymbol{\theta}_{z}\right)=\mathcal{M}\left(v_{z_{j}} \mid \boldsymbol{\theta}_{z}\right)
\end{array} .\right.
$$

Depending on the choice of the $\mathcal{M}$ distribution, a different prior corresponds to $p\left(z_{j} \mid 0, \boldsymbol{\theta}_{z}\right)$. In the following, a brief presentation of the $\mathcal{M}$ distributions considered and their corresponding heavy-tailed prior distribution is given.

\section{1. $\mathcal{I G}$ mixing distribution $-\mathcal{S} t$ prior}

Inverse Gamma is considered as the mixing distribution in Eq. (3), $\mathcal{M}\left(v_{z_{j}} \mid \boldsymbol{\theta}_{z}\right)=\mathcal{I} \mathcal{G}\left(v_{z_{j}} \mid \alpha_{z}, \beta_{z}\right)$. The corresponding hyperparameters are in this case $\boldsymbol{\theta}_{z}=\left(\alpha_{z}, \beta_{z}\right)$ and the corresponding marginal of the joint probability distribution $\left(v_{z_{j}} \mid \alpha_{z}, \beta_{z}\right)$ is a two-parameter $\mathcal{S} t$ distribution:

$$
p\left(z_{j} \mid \alpha_{z}, \beta_{z}\right)=\frac{\Gamma\left(\alpha_{z}+\frac{1}{2}\right)}{\sqrt{2 \pi \beta_{z}} \Gamma\left(\alpha_{z}\right)}\left(1+\frac{z_{j}^{2}}{2 \beta_{z}}\right)^{-\left(\alpha_{z}+\frac{1}{2}\right)} .
$$

Observation: Imposing $\alpha_{z}=\beta_{z}=\nu_{z} / 2$ in the $\mathcal{I} \mathcal{G}$ mixing distribution leads to the standard $\mathcal{S} t$ form with one parameter [5].

\section{2. $\mathcal{G} \mathcal{I} \mathcal{G}$ mixing distribution $-\mathcal{N} \mathcal{I} \mathcal{G}$ prior}

In Eq. (3) the generalized inverse Gaussian is considered as the mixing distribution, $\mathcal{M}\left(v_{z_{j}} \mid \boldsymbol{\theta}_{z}\right)=\mathcal{G I G}\left(v_{z_{j}} \mid \gamma_{z}^{2}, \delta_{z}^{2},-1 / 2\right)$. The hyperparameters are $\boldsymbol{\theta}_{z}=\left(\gamma_{z}^{2}, \delta_{z}^{2}\right)$ and the corresponding marginal of the joint probability distribution $\left(v_{z_{j}} \mid \gamma_{z}^{2}, \delta_{z}^{2}\right)$ is a zero location and asymmetry parameter $\mathcal{N} \mathcal{I} \mathcal{G}$ distribution:

$$
p\left(z_{j} \mid \gamma_{z}, \delta_{z}\right)=\frac{\gamma_{z} \delta_{z} \mathcal{K}_{1}\left(\gamma_{z} \sqrt{\delta_{z}^{2}+z_{j}^{2}}\right)}{\pi \sqrt{\delta_{z}^{2}+z_{j}^{2}}} \exp \left\{\gamma_{z} \delta_{z}\right\}
$$

where $\mathcal{K}_{1}$ denotes the modified Bessel function of the second kind.

\section{3. $\mathcal{G}$ mixing distribution $-\mathcal{V} \mathcal{G}$ prior}

In Eq. (3) Gamma is considered as the mixing distribution, $\mathcal{M}\left(v_{z_{j}} \mid \boldsymbol{\theta}_{z}\right)=\mathcal{G}\left(v_{z_{j}} \mid k_{z}, \theta_{z}\right)$. In this case $\boldsymbol{\theta}_{z}=\left(k_{z}, \theta_{z}\right)$ and the joint probability distribution marginal is a zero location and asymmetry parameter Variance-Gamma distribution:

$$
p\left(z_{j} \mid k_{z}, \theta_{z}\right)=\frac{\theta_{z}^{2 k_{z}}\left|z_{j}\right|^{k_{z}-\frac{1}{2}} \mathcal{K}_{k_{z}-\frac{1}{2}}\left(k_{z}\left|z_{j}\right|\right)}{\sqrt{\pi} \Gamma\left(k_{z}\right)\left(2 \theta_{z}\right)^{k_{z}-\frac{1}{2}}} .
$$

Observation: A particular case of the $\mathcal{V G}$ prior is the Laplace prior, corresponding to Exponential mixing distribution [6]. Observation: $\mathcal{G} \mathcal{I} \mathcal{G}(x \mid 2 \theta, \delta \searrow 0, k)=\mathcal{G}(x \mid k, \theta) ; \mathcal{V G}$ prior can also be viewed as a Normal variance mixture with $\mathcal{G I} \mathcal{G}$ mixing distribution.

\section{JMAP ESTIMATION}

JMAP estimates the unknowns of the hierarchical model by maximizing the posterior distribution, Eq. (??) or minimizing the criterion $\mathcal{L}$, defined by

$$
\mathcal{L}\left(\boldsymbol{f}, \boldsymbol{z}, \boldsymbol{v}_{\xi}, \boldsymbol{v}_{\epsilon}, \boldsymbol{v}_{z}\right)=-\ln p\left(\boldsymbol{f}, \boldsymbol{z}, \boldsymbol{v}_{\xi}, \boldsymbol{v}_{\epsilon}, \boldsymbol{v}_{z} \mid \boldsymbol{g}\right)
$$

For Normal variance mixture priors, the structure of the hierarchical model, Fig. (1) is the same for $p\left(\boldsymbol{g} \mid \boldsymbol{f}, \boldsymbol{v}_{\epsilon}\right), p\left(\boldsymbol{f} \mid \boldsymbol{z}, \boldsymbol{v}_{\xi}\right)$ and $p\left(\boldsymbol{z} \mid \boldsymbol{v}_{z}\right)$ so the first part of the posterior distribution (and the criterion $\mathcal{L}$ ) is the same, regardless of the choice of the $\mathcal{M}$. Consequently, the estimates $\widehat{\boldsymbol{f}}$ and $\widehat{\boldsymbol{z}}$ have the same analytical expressions. The minimization of the criterion $\mathcal{L}$ by alternate optimization with respect to each unknown gives the analytical expression:

$$
\begin{aligned}
& \widehat{\boldsymbol{f}}=\left(\boldsymbol{H}^{T} \widehat{\boldsymbol{V}}_{\epsilon}^{-1} \boldsymbol{H}+\widehat{\boldsymbol{V}}_{\xi}^{-1}\right)^{-1}\left(\boldsymbol{H}^{T} \widehat{\boldsymbol{V}}_{\epsilon}^{-1} \boldsymbol{g}+\widehat{\boldsymbol{V}}_{\xi}^{-1} \boldsymbol{D} \widehat{\boldsymbol{z}}\right) \\
& \widehat{\boldsymbol{z}}=\left(\boldsymbol{D}^{T} \widehat{\boldsymbol{V}}_{\xi}^{-1} \boldsymbol{D}+\widehat{\boldsymbol{V}}_{z}^{-1}\right)^{-1} \boldsymbol{D}^{T} \widehat{\boldsymbol{V}}_{\xi}^{-1} \widehat{\boldsymbol{f}}
\end{aligned}
$$

where

$$
\begin{aligned}
\widehat{\boldsymbol{v}}_{\xi} & =\left[\ldots \widehat{v}_{\xi_{j}} \ldots\right] ; \widehat{\boldsymbol{v}}_{\epsilon}=\left[\ldots \widehat{v}_{\epsilon_{i}} \ldots\right] ; \widehat{\boldsymbol{v}}_{z}=\left[\ldots \widehat{v}_{z_{j}} \ldots\right] \\
\widehat{\boldsymbol{V}}_{\xi} & =\operatorname{diag}\left[\widehat{\boldsymbol{v}}_{\xi}\right] ; \widehat{\boldsymbol{V}}_{\epsilon}=\operatorname{diag}\left[\widehat{\boldsymbol{v}}_{\epsilon}\right] ; \widehat{\boldsymbol{V}}_{z}=\operatorname{diag}\left[\widehat{\boldsymbol{v}}_{z}\right]
\end{aligned}
$$

Introducing the notations

$$
d\left(\xi_{j}\right)=\widehat{f}_{j}-\boldsymbol{D}_{j} \boldsymbol{z} ; d\left(\epsilon_{i}\right)=g_{i}-\boldsymbol{H}_{i} \boldsymbol{f} ; d\left(z_{j}\right)=z_{j}
$$

where $f_{j}$ denotes the $j^{\text {th }}$ element of $\boldsymbol{f}, \boldsymbol{D}_{j}$ denotes the $j^{\text {th }}$ line of $\boldsymbol{D}, g_{i}$ denotes the $i^{t h}$ element of $\boldsymbol{g}$ and $\boldsymbol{H}_{i}$ denotes the $i^{t h}$ line of $\boldsymbol{H}$, the analytical expressions of variance estimates 
depending on mixing distribution are:

$$
\begin{aligned}
\text { St: } \widehat{v}_{\zeta_{k}} & =\frac{\beta_{\zeta}+\frac{1}{2} d^{2}\left(\zeta_{k}\right)}{\alpha_{\zeta}+\frac{3}{2}} \\
\text { NIG: } \widehat{v}_{\zeta_{k}} & =\frac{\sqrt{4+\gamma_{\zeta}^{2}\left(\delta_{\zeta}^{2}+d^{2}\left(\zeta_{k}\right)\right)}-2}{\gamma_{\zeta}^{2}} \\
\text { VG: } \widehat{v}_{\zeta_{k}} & =\frac{\sqrt{\left(\frac{3}{2}-k_{\zeta}\right)^{2}+2 \frac{1}{\theta_{\zeta}} d^{2}\left(\zeta_{k}\right)}-\left(\frac{3}{2}-k_{\zeta}\right)}{2 \frac{1}{\theta_{\zeta}}}
\end{aligned}
$$

where $\zeta=\{\xi, \epsilon, z\}$ The JMAP iterative algorithms are presented in Fig (2).

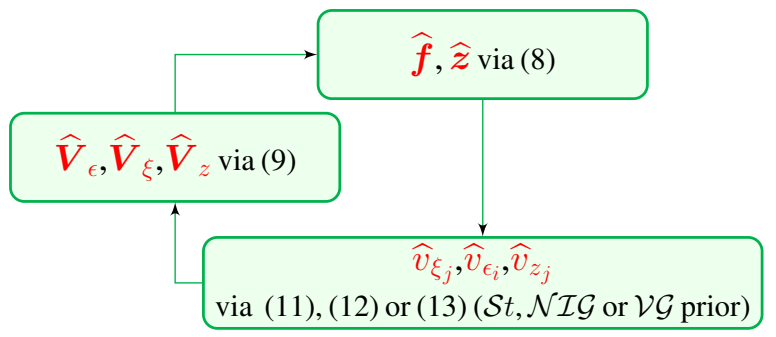

Fig. 2: Joint MAP iterative algorithm

\section{SIMULATION RESULTS}

$\mathrm{X}$-ray CT piecewise constant images reconstruction problem is addressed in this section. Results are reported for 2D and $3 \mathrm{D}$ simulations. The Shepp Logan phantom $\left(256^{2}\right.$ respectively $256^{3}$ ) is used as the original image. The projections are simulated uniformly between $0^{\circ}$ and $180^{\circ}$. The sparse representation of the image is obtained via the multilevel Haar transform. For 3D simulations, GPU is used. The normalised mean square error (NMSE) is used for estimating the reconstruction deviations. The results correspond for 50 iteration. In the context of X-ray CT, the matrix $\boldsymbol{H}$ is not considered explicitly but via the forward projection $\boldsymbol{H} \boldsymbol{f}$ and the backprojection $\boldsymbol{H}^{t} \boldsymbol{g}$ operators. The estimations corresponding to $f$ and $z$ Eq. (8) are computed using the gradient descent algorithm, and the descent step length is obtained using an optimized step length strategy [3].

We report simulations results for $128,64,32$ projections and noise levels corresponding to $\mathrm{SNR}=\{40,30,20,10\} \mathrm{dB}$, for which the normalized mean reconstruction error (NME) $\frac{\|\boldsymbol{f}-\widehat{\boldsymbol{f}}\|^{2}}{\|\boldsymbol{f}\|}$ is computed (Table (1) and (2)). For the 2D case, the sensibility of the algorithms to the hyperparameters is investigated for all cases of levels of noise and numbers of projections by considering for each hyperparameter $\{0.001,0.01$, $0.1,1,2.1,10,100\}$.

Figure (3) presents the evolution of the NME during the iterations for the three JMAP algorithms depending on the hyperparameters, for $40 \mathrm{~dB}$ (left column) and $30 \mathrm{~dB}$ (right column). The $\mathcal{S} t$ prior presents a strong sensibility with

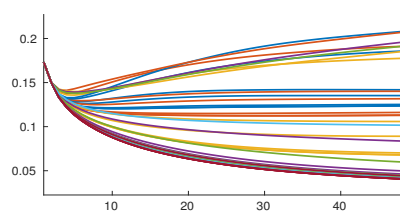

(a) St. 32 proi.. $40 \mathrm{~dB}$

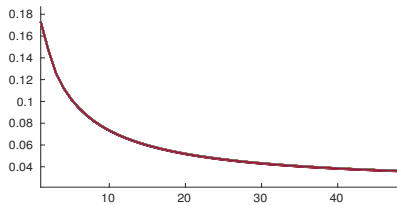

(c) $\mathcal{N} \mathcal{I} G .32$ proi. $.40 \mathrm{~dB}$

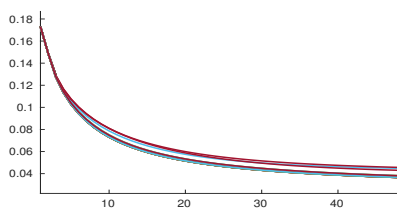

(e) $\mathcal{V G}, 32$ proj., $40 \mathrm{~dB}$

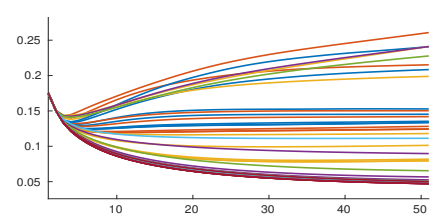

(b) St. 32 proi.. $30 \mathrm{~dB}$

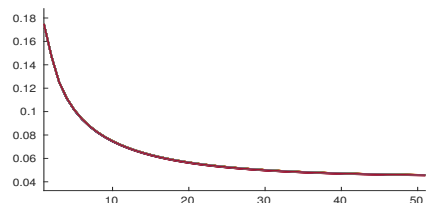

(d) $\mathcal{N} \mathcal{I} G .32$ proi. . $30 \mathrm{~dB}$

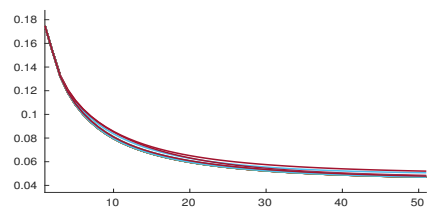

(f) $\mathcal{V G}, 32$ proj., $30 \mathrm{~dB}$
Fig. 3: 2D study: JMAP algorithms reconstruction error (NME) (y axis) during iterations ( $\mathrm{x}$ axis) depending on the hyperparameters.

respect to the hyperparameters, Figure (3a) and (3b), with very different reconstruction performances. For the $\mathcal{V G}$ prior, the influence of the hyperparameters is week, Figure (3c) and (3d). For the $\mathcal{N} \mathcal{I} \mathcal{G}$ prior the reconstruction performances depending on the hyperparameters are almost superposed, Figure (3e) and (3f). A comparison between the three priors reconstruction NMEs during iterations, corresponding to the experimental optimal hyperparameters is presented in Figure (4) for $40 \mathrm{~dB}$ (left) and $30 \mathrm{~dB}$ (right) for 64 and 32 projections.

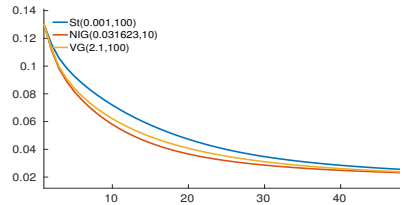

(a) 64 proi.. $40 \mathrm{~dB}$

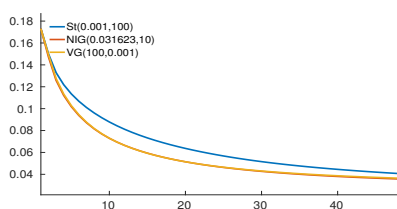

(c) 32 proj., $40 \mathrm{~dB}$

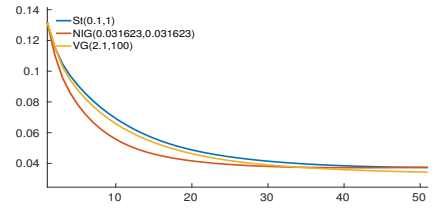

(b) 64 proi...30dB

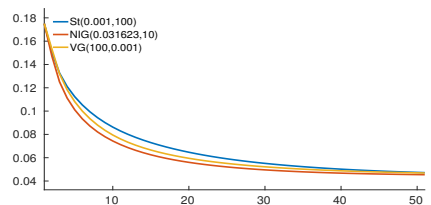

(d) 32 proj., $30 \mathrm{~dB}$
Fig. 4: 2D study: JMAP algorithms NME (y axis) for the optimal hyperparameters duiring iterations ( $\mathrm{x}$ axis), $40 \mathrm{~dB}$ and $30 \mathrm{~dB}$. 


\begin{tabular}{|c|c|c|c|c|c|c|c|c|c|}
\hline \multirow{3}{*}{$\begin{array}{c}\text { 2D } \\
\text { SNR } \\
\text { Prior }\end{array}$} & \multicolumn{9}{|c|}{ phantom size: $256 * 256$} \\
\hline & \multicolumn{3}{|c|}{$40 \mathrm{~dB}$} & \multicolumn{3}{|c|}{$30 \mathrm{~dB}$} & \multicolumn{3}{|c|}{$20 \mathrm{~dB}$} \\
\hline & $\overline{\mathcal{S} t}$ & $\overline{N \mathcal{I G}}$ & $\overline{\mathcal{V G}}$ & $\mathcal{S} t$ & $\mathcal{N \mathcal { I G }}$ & $\overline{\mathcal{V G}}$ & $\overline{\mathcal{S} t}$ & $\mathcal{N \mathcal { I G }}$ & $\overline{\mathcal{V G}}$ \\
\hline 128 proj & 0.0124 & 0.0118 & 0.0127 & 0.0271 & 0.0311 & 0.0279 & 0.0833 & 0.1923 & 0.1561 \\
\hline 64proj & 0.0247 & 0.0227 & 0.0231 & 0.0373 & 0.0375 & 0.0343 & 0.1099 & 0.1724 & 0.1368 \\
\hline 32 proj & 0.0396 & 0.0351 & 0.0358 & 0.0472 & 0.0454 & 0.0469 & 0.1163 & 0.1415 & 0.1186 \\
\hline
\end{tabular}

Table 1: 2D case: NMSE of reconstructed phantom with 50 iterations corresponding to the three priors.

\begin{tabular}{|c|c|c|c|c|c|c|c|c|c|}
\hline \multirow{3}{*}{$\begin{array}{c}\text { 3D } \\
\text { SNR } \\
\text { Prior }\end{array}$} & \multicolumn{9}{|c|}{ phantom size: $256 * 256 * 256$} \\
\hline & \multicolumn{3}{|c|}{$40 \mathrm{~dB}$} & \multicolumn{3}{|c|}{$30 \mathrm{~dB}$} & \multicolumn{3}{|c|}{$20 \mathrm{~dB}$} \\
\hline & $\mathcal{S} t$ & $\overline{N \mathcal{I G}}$ & $\mathcal{V G}$ & $\mathcal{S} t$ & $\overline{N \mathcal{I G}}$ & $\mathcal{V G}$ & $\mathcal{S} t$ & $\mathcal{N \mathcal { I } G}$ & $\mathcal{V G}$ \\
\hline 128proj & 0.0744 & 0.0112 & 0.0326 & 0.0330 & 0.0309 & 0.0580 & 0.1031 & 0.1618 & 0.1451 \\
\hline 64 proj & 0.0783 & 0.0175 & 0.0379 & 0.0552 & 0.0352 & 0.0623 & 0.1086 & 0.1517 & 0.1424 \\
\hline 32 proj & 0.1283 & 0.0275 & 0.0357 & 0.1059 & 0.0406 & 0.0575 & 0.1219 & 0.1274 & 0.1258 \\
\hline
\end{tabular}

Table 2: 3D case: NMSE of reconstructed phantom with 50 iterations and computation time (s) per iteration.

The 2D reconstruction performances for all levels of noise and number of projections considered, corresponding to the optimal hyperparameters are presented in Table (1). A different behaviour of the priors is reported depending on the level of noise. For $40 \mathrm{~dB}$ and $30 \mathrm{~dB}$ the reconstruction performances are very similar. In particular, the $\mathcal{N} \mathcal{I} \mathcal{G}$ seems to give slightly better performances for $40 \mathrm{~dB}$, for all cases of number of projections considered. However, for important levels of noise, $20 \mathrm{~dB}$, the reconstruction performances seems to be significantly better for the $\mathcal{S} t$ prior. The reconstructed 2D Shepp Logan phantom corresponding to the three priors is presented in Figure (5): 64 proj and 40dB in Figure (5a) and 32 proj and $20 \mathrm{~dB}$ in Figure $(5 \mathrm{~b})$.
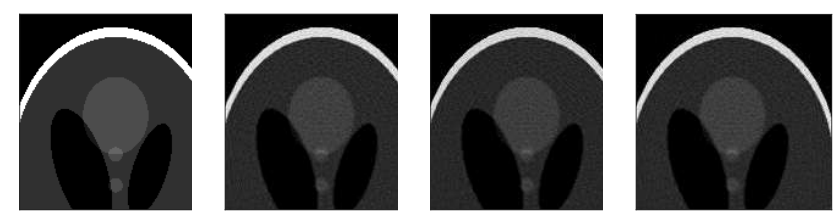

(a) 2D: Original. St. $\mathcal{N} \mathcal{I G} . \mathcal{V G}(64$ proi. $40 \mathrm{~dB})$
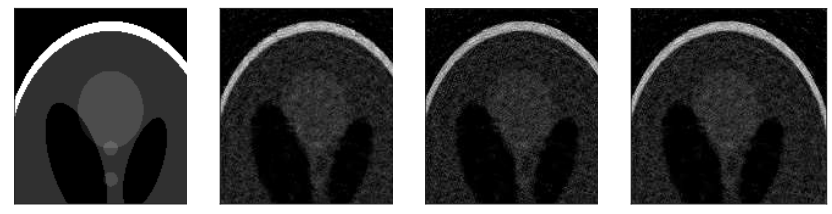

(b) 2D: Original, $\mathcal{S} t, \mathcal{N} \mathcal{I} \mathcal{G}, \mathcal{V G}$ (32 proj, 20dB)

Fig. 5: 2D reconstructions corresponding to $\mathcal{S} t, \mathcal{N} \mathcal{I} \mathcal{G}$ and $\mathcal{V G}$

The 3D reconstruction performances for all levels of noise and number of projections considered, corresponding to the optimal hyperparameters are presented in Table (2). In the $3 \mathrm{D}$ case, the $\mathcal{N} \mathcal{I} \mathcal{G}$ seems to give better reconstruction performances for $40 \mathrm{~dB}$ and $30 \mathrm{~dB}$ while the $\mathcal{S} t$ prior seems to be more appropriate for $20 \mathrm{~dB}$. The middle slice of reconstructed 3D Shepp Logan phantom corresponding to the three priors is presented in Figure (6): 64 proj and 40dB in Figure (6a) and 32 proj and $20 \mathrm{~dB}$ in Figure $(6 \mathrm{~b})$.
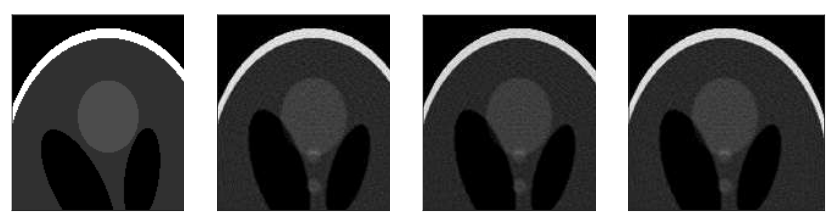

(a) 3D middle slice: Original. $S t . \mathcal{N} \mathcal{I G}$. VG $(64$ nroi. $40 \mathrm{~dB})$
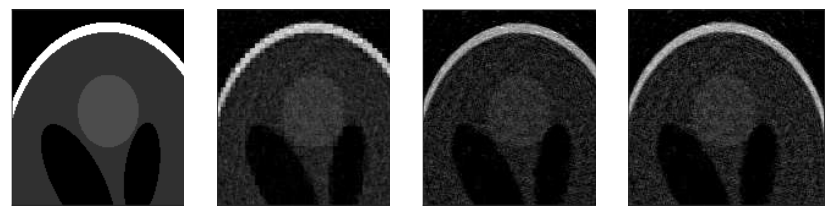

(b) 3D middle slice: Original, $\mathcal{S} t, \mathcal{N} \mathcal{I} \mathcal{G}, \mathcal{V G}$ (32 proj, 20dB)

Fig. 6: $3 \mathrm{D}$ reconstructions corresponding to $\mathcal{S} t, \mathcal{N} \mathcal{I} \mathcal{G}$ and $\mathcal{V G}$

\section{CONCLUSION}

In this paper we have presented the JMAP algorithms corresponding to three different prior models in the sparsity enforcing context. We have considered heavy tailed distribution expressed as Normal variance mixture, showing that essentially the updating equations have the same analytical expression, except for the variances appearing in the hierarchical model. First we have showed different behaviours of the priors in terms of sensibility to the hyperparameters. Then we have examined the reconstruction performances for $2 \mathrm{D}$ and $3 \mathrm{D}$, Table (1) and (2) reporting that for different levels of noise different priors seem to give better performances. A study considering simulations results corresponding to other phantoms and real tomographic data is currently in progress. A more detailed study considering the sparsity rate associated with each prior depending on the hyperparameters needs 
to be developed in order to investigate the reconstruction results corresponding to such hyperparameters. Also, the very similar posterior mean estimation via Variational Bayesian Approximation (VBA) are to be investigated and compared. The main conclusion of this study is that for high SNR values the $\mathcal{N} \mathcal{I} \mathcal{G}$ prior is very stable and gives slightly better performances while for lower SNR values the $\mathcal{S} t$ model has better performances in terms of reconstruction.

\section{REFERENCES}

[1] Saman Atapattu, Chintha Tellambura, and Hai Jiang. A mixture gamma distribution to model the snr of wireless channels. IEEE Transactions on Wireless Communications, 10:4193-4203, 2011.

[2] S. D. Babacan, S. Nakajima, and M. N. Do. Bayesian group-sparse modeling and variational inference. IEEE Trans. Signal Process, 14:2906-2921, 2014.

[3] Stephen Boyd and Lieven Vandenberghe. Convex optimization. Cambridge university press, 2004.

[4] M. Dumitru, A. Mohammad-Djafari, and Baghai S. S. Precise periodic components estimation for chronobiological signals through bayesian inference with sparsity enforcing prior. EURASIP Journal on Bioinformatics and Systems Biology, Springer, 3, 2016.

[5] Mircea Dumitru. A Bayesian approach for periodic components estimation for chronobiological signals. $\mathrm{PhD}$ thesis, Université Paris-Saclay, Mar 2016.

[6] T. Eltoft, Taesu Kim, and Te-Won Lee. On the multivariate laplace distribution. IEEE Signal Processing Letters, 13:300-303, 2006.

[7] Samuel Kotz and Saralees Nadarajah. Multivariate TDistributions and Their Applications. Cambridge University Press, 2010.

[8] Ali Mohammad-Djafari. Bayesian approach with prior models which enforce sparsity in signal and image processing. EURASIP Journal on Advances in Signal Processing, 52, 2012.

[9] Ali Mohammad-Djafari and Dumitru Mircea. Bayesian sparse solutions to linear inverse problems with nonstationary noise with student-t priors. Digital Signal Processing, 47:128-156, 2015.

[10] Tor Arne Oigard and Alfred Hanssen. The multivariate normal inverse gaussian heavy-tailed distribution; simulation and estimation. pages 1489-1492. IEEE, 2002.

[11] Tor Arne Oigard, Alfred Hanssen, and Roy Edgar Hansen. The multivariate normal inverse gaussian distribution: Em-estimation and analysis of synthetic aperture sonar data. pages 1433-1436. IEEE, 2004.
[12] H. Sadreazami, M. Omair Ahmad, and M. N. S. Swamy. Contourlet domain image denoising using normal inverse gaussian distribution. IEEE, 2014.

[13] Rasool Tahmasbi and Sadegh Rezaei. A soft voice activity detection using garch filter and variance gamma distribution. IEEE Transactions on Audio, Speech and Language Processing, 15:1129-1134, 2007.

[14] Konstantinos E. Themelis, Athanasios A. Rontogiannis, and Konstantinos D. Koutroumbas. A variational bayes framework for sparse adaptive estimation. IEEE Transactions on Signal Processing, 62:4723-4736, 2014.

[15] Li Wang, Ali Mohammad-Djafari, Nicolas Gac, and Mircea Dumitru. Computed tomography reconstruction based on a hierarchical model and variational Bayesian method. In 2016 IEEE Int. Conf. on Acoustics, Speech and Signal Processing (ICASSP), pages 883-887. IEEE, 2016. 